\title{
Dressed Feshbach Molecules in the BEC-BCS Crossover
}

\begin{abstract}
M. W. J. Romans and H. T. C. Stoof
Institute for Theoretical Physics, Utrecht University, Leuvenlaan 4, 3584 CE Utrecht, The Netherlands (Received 13 June 2005; revised manuscript received 23 September 2005; published 28 December 2005)

We present the random phase approximation (RPA) theory of the Bose-Einstein-condensationBardeen-Cooper-Schrieffer crossover in an atomic Fermi gas near a Feshbach resonance that includes the relevant two-body atomic physics exactly. This allows us to determine the probability $Z$ for the dressed molecules in the Bose-Einstein condensate to be in the closed channel of the Feshbach resonance and to compare with the recent experiments of Partridge et al. [95, 020404 (2005)] with ${ }^{6} \mathrm{Li}$, who have measured the same quantity.
\end{abstract}

DOI: $10.1103 /$ PhysRevLett.95.260407

PACS numbers: 03.75. $-\mathrm{b}, 67.40 .-\mathrm{w}, 39.25 .+\mathrm{k}$

Introduction. - The superfluid phase in an atomic Fermi gas near a Feshbach resonance realizes a fundamentally new state of matter, which shows a macroscopic coherence between atom pairs and molecules that is controlled by the applied magnetic field. As a result, such a gas offers the exciting possibility to study in great detail the crossover between the Bose-Einstein condensation (BEC) of diatomic molecules and the Bose-Einstein condensation of atomic Cooper pairs, i.e., the Bardeen-Cooper-Schrieffer (BCS) transition [1-4]. In fact, the BEC-BCS crossover region is presently already actively being explored by a number of experimental groups around the world [5-10].

In more detail the physics of the BEC-BCS crossover occurring near a Feshbach resonance can be understood as follows: the superfluid phase of the gas is always associated with a Bose-Einstein condensate of pairs, but the wave function of the pairs or dressed molecules is given by the linear superposition

$$
\left.\left.\left\langle\mathbf{r} \mid \chi_{\text {dressed }}\right\rangle=\sqrt{Z} \chi_{\mathrm{m}}(\mathbf{r}) \mid \text { closed }\right\rangle+\sqrt{1-Z} \chi_{\text {aa }}(\mathbf{r}) \mid \text { open }\right\rangle,
$$

where $\mathbf{r}$ denotes the interatomic distance. In the BEC limit the bare molecular energy level lies far below the threshold of the two-atom continuum and we have $Z \simeq 1$. In that case we are dealing with a Bose-Einstein condensate of tightly bound diatomic molecules and the spatial part of the pair wave function is equal to the bare molecular wave function $\chi_{\mathrm{m}}(\mathbf{r})$. The spin part of the pair wave function is then equal to $\mid$ closed $\rangle$, i.e., the spin state of the closed channel that causes the Feshbach resonance [11]. In the BCS limit the bare molecular energy level lies far above the threshold of the two-atom continuum and can be adiabatically eliminated. We then have that $Z \simeq 0$ and the spatial part of the pair wave function equals the usual BCS wave function for atomic Cooper pairs $\chi_{\text {aa }}(\mathbf{r})$. This Cooper-pair wave function depends also on the magnetic field, because the effective attraction between the atoms after the adiabatic elimination of the bare molecular state depends on the energy of that state. The spin state of the Cooper pairs is, however, always equal to the spin state |open $\rangle$ of the open channel of the Feshbach problem.
The probability $Z$ therefore plays a crucial role in the description of the BEC-BCS crossover since it quantifies the mixing between the atom pairs and the bare molecules in the gas. Unfortunately, however, the various theories $[12-20]$ that are presently being used to understand the outcome of the experiments are unable to accurately determine this quantity. This comes about because either $Z$ is assumed to be zero from the outset, the many-body theory does not incorporate the relevant two-body Feshbach physics exactly, or the theory is able to determine only the total number of bare molecules in the gas and thus requires an additional assumption about the total number of dressed molecules to extract $Z$. This situation is particularly unsettling because of the recent ${ }^{6} \mathrm{Li}$ experiments of Partridge et al. [10], which have used the photoassociation rate to measure the value of $Z$ throughout the crossover regime. In view of the above situation it is pressing to develop an $a b$ initio many-body theory for the calculation of $Z$. How that may be achieved is the main topic of this Letter.

$B E C-B C S$ crossover theory. - Introducing creation and annihilation operators for the bare molecules and atoms, the effective grand-canonical Hamiltonian of the gas with chemical potential $\mu$ becomes $[11,21,22]$

$$
\begin{aligned}
H= & \int d \mathbf{x} \psi_{\mathrm{m}}^{\dagger}(\mathbf{x})\left(-\frac{\hbar^{2} \boldsymbol{\nabla}^{2}}{4 m}+\delta-2 \mu\right) \psi_{\mathrm{m}}(\mathbf{x}) \\
& +\sum_{\sigma=\uparrow, \downarrow} \int d x \psi_{\sigma}^{\dagger}(\mathbf{x})\left(-\frac{\hbar^{2} \boldsymbol{\nabla}^{2}}{2 m}-\mu\right) \psi_{\sigma}(\mathbf{x}) \\
& +g \int d x\left[\psi_{\mathrm{m}}^{\dagger}(\mathbf{x}) \psi_{\uparrow}(\mathbf{x}) \psi_{\downarrow}(\mathbf{x})+\psi_{\downarrow}^{\dagger}(\mathbf{x}) \psi_{\uparrow}^{\dagger}(\mathbf{x}) \psi_{\mathrm{m}}(\mathbf{x})\right] \\
& +\frac{4 \pi a_{\mathrm{bg}} \hbar^{2}}{m} \int d x \psi_{\downarrow}^{\dagger}(\mathbf{x}) \psi_{\uparrow}^{\dagger}(\mathbf{x}) \psi_{\uparrow}(\mathbf{x}) \psi_{\downarrow}(\mathbf{x}),
\end{aligned}
$$

where the two hyperfine states of the atoms are denoted by $|\uparrow\rangle$ and $|\downarrow\rangle$, and the magnetic-moment difference $\Delta \mu_{\text {mag }}$ between the hyperfine states $\mid$ closed $\rangle$ and $\mid$ open $\rangle \equiv(|\uparrow \downarrow\rangle-$ $|\downarrow \uparrow\rangle) / \sqrt{2}$ gives the so-called detuning from resonance $\delta=$ $\Delta \mu_{\text {mag }}\left(B-B_{0}\right)$. The atomic mass is given by $m$. Note that the atom-molecule coupling constant $g$ and the background scattering length $a_{\mathrm{bg}}$ depend on the magnetic field $B$ in 
such a manner that the total scattering length $a=a_{\mathrm{bg}}-$ $m g^{2} / 4 \pi \hbar^{2} \delta$ agrees with the Feshbach resonance of interest. This is especially important for the broad Feshbach resonance near 834 Gauss that is used in all ${ }^{6} \mathrm{Li}$ experiments up to date $[23,24]$.

From now on we consider only the most interesting region close to resonance, where $a_{\mathrm{bg}} \ll a$ and the effective interaction between the atoms is dominated by the resonant part $-g^{2} / \delta$. This suggests that the last term in the righthand side of Eq. (1) can be neglected altogether. This is, however, not true because we can neglect this term only after we have included its effect on the atom-molecule coupling [25]. Physically, the reason for this subtlety is that the above Hamiltonian is an effective Hamiltonian that is only valid for low energies. However, to properly account for the two-body physics near a Feshbach resonance high-energy states are also needed. The main effect of these high-energy states is to renormalize the atommolecule coupling to $g(\mathbf{k}) \equiv g /\left(1+i k a_{\mathrm{bg}}\right)$, where $\mathbf{k}$ is the relative momentum of the atoms involved in the cou- pling $[11,25]$. Only after having performed this substitution are we allowed to neglect the background interaction between the atoms. Using standard functional methods the fermionic fields can then be integrated out exactly. This leads to an effective action for the molecules that at sufficiently low temperatures has a minimum at a nonzero value of $\left\langle\psi_{\mathrm{m}}(\mathbf{x})\right\rangle \equiv \sqrt{Z n_{\mathrm{mc}}}$, where we introduced the dressed molecular condensate density $n_{\mathrm{mc}}$. Neglecting fluctuations at this point leads to a mean-field theory of the BEC-BCS crossover. As mentioned in the introduction, however, this mean-field theory is unable to calculate the probability $Z$ since it only determines the bare molecular condensate density $\left|\left\langle\psi_{\mathrm{m}}(\mathbf{x})\right\rangle\right|^{2}$. We therefore also consider the quadratic fluctuations around the minimum of the effective molecular action, i.e., we consider the Bogoliubov theory of the bare molecules.

The Gaussian fluctuations are determined by normal and anomalous self-energies of the bare molecules that are obtained from integrating out the fermions. They contain the BCS propagators, and at zero temperature reduce to

$$
\begin{aligned}
& \hbar \Sigma_{11}(\mathbf{k}, i \omega)=\int \frac{d \mathbf{k}^{\prime}}{(2 \pi)^{3}}\left|g\left(\mathbf{k}^{\prime}\right)\right|^{2}\left\{\frac{\left|u_{\mathrm{a}}\left(\mathbf{k}_{+}^{\prime}\right)\right|^{2}\left|u_{\mathrm{a}}\left(\mathbf{k}_{-}^{\prime}\right)\right|^{2}}{i \hbar \omega-\hbar \omega_{\mathrm{a}}\left(\mathbf{k}_{+}^{\prime}\right)-\hbar \omega_{\mathrm{a}}\left(\mathbf{k}_{-}^{\prime}\right)}-\frac{\left|v_{\mathrm{a}}\left(\mathbf{k}_{+}^{\prime}\right)\right|^{2}\left|v_{\mathrm{a}}\left(\mathbf{k}_{-}^{\prime}\right)\right|^{2}}{i \hbar \omega+\hbar \omega_{\mathrm{a}}\left(\mathbf{k}_{+}^{\prime}\right)+\hbar \omega_{\mathrm{a}}\left(\mathbf{k}_{-}^{\prime}\right)}+\frac{1}{2 \epsilon\left(\mathbf{k}^{\prime}\right)}\right\}, \\
& \hbar \Sigma_{12}(\mathbf{k}, i \omega)=2 \int \frac{d \mathbf{k}^{\prime}}{(2 \pi)^{3}}\left|g\left(\mathbf{k}^{\prime}\right)\right|^{2}\left\{u_{\mathrm{a}}\left(\mathbf{k}_{+}^{\prime}\right) v_{\mathrm{a}}\left(\mathbf{k}_{+}^{\prime}\right) u_{\mathrm{a}}\left(\mathbf{k}_{-}^{\prime}\right) v_{\mathrm{a}}\left(\mathbf{k}_{-}^{\prime}\right) \frac{\hbar \omega_{\mathrm{a}}\left(\mathbf{k}_{+}^{\prime}\right)+\hbar \omega_{\mathrm{a}}\left(\mathbf{k}_{-}^{\prime}\right)}{\left[\hbar \omega_{\mathrm{a}}\left(\mathbf{k}_{+}^{\prime}\right)+\hbar \omega_{\mathrm{a}}\left(\mathbf{k}_{-}^{\prime}\right)\right]^{2}+(\hbar \omega)^{2}}\right\} .
\end{aligned}
$$

Here we have also introduced the BCS dispersion $\hbar \omega_{\mathrm{a}}(\mathbf{k})=\sqrt{(\epsilon(\mathbf{k})-\mu)^{2}+|g(\mathbf{k})|^{2} Z n_{\mathrm{mc}}}$, the bare atomic dispersion $\epsilon(\mathbf{k})=\hbar^{2} \mathbf{k}^{2} / 2 m$, the usual BCS coherence factors $u_{\mathrm{a}}(\mathbf{k})$ and $v_{\mathrm{a}}(\mathbf{k})$, and the notation $\mathbf{k}_{ \pm}^{\prime}=$ $\mathbf{k} / 2 \pm \mathbf{k}^{\prime}$. In terms of the above self-energies the minimum of the effective action is determined by the exact Hugenholtz-Pines relation $2 \mu=\delta+\hbar \Sigma_{11}(\mathbf{0}, 0)-$ $\hbar \Sigma_{12}(\mathbf{0}, 0)$ [26], which turns out to be equal to a modified BCS gap equation

$$
\delta-2 \mu=\int \frac{d \mathbf{k}}{(2 \pi)^{3}}|g(\mathbf{k})|^{2}\left(\frac{1}{2 \hbar \omega_{\mathrm{a}}(\mathbf{k})}-\frac{1}{2 \epsilon(\mathbf{k})}\right) .
$$

Finally, we also need the equation of state, which we for consistency reasons [11] obtain by differentiating the thermodynamic potential with respect to the chemical potential. Including the effect of the fluctuations we obtain for the total density of atoms

$$
n=\operatorname{Tr}\left[G_{\mathrm{a}} \sigma_{3}\right]+2 Z n_{\mathrm{mc}}-\operatorname{Tr}\left[G_{\mathrm{m}}\right]+\frac{1}{2} \operatorname{Tr}\left[G_{\mathrm{m}} \frac{\partial \hbar \Sigma}{\partial \mu}\right],
$$

where $G_{\mathrm{a}}$ and $G_{\mathrm{m}}$ are the Nambu $(2 \times 2)$-matrix Green's functions of the bare atoms and molecules, respectively, $\sigma_{3}$ is the third Pauli matrix, and $\Sigma$ stands for the self-energy matrix of the molecules. For a given density and magnetic field the last two equations determine only the bare molecular condensate density and the chemical potential. Hence, we need a third equation to determine also $Z$.
Dressed molecules. - Let us first discuss the physics behind the equation of state in Eq. (4). The first two terms represent the mean-field theory without fluctuations that is most often used in the recent literature [14-16,18]. Because of the absence of fluctuations all the molecules are Bose-Einstein condensed and there is no depletion. The third term precisely corresponds to this depletion. Finally, the fourth term physically describes the dressing of the bare atoms and molecules. This can be made more clear by reformulating the equation of state in terms of dressed atoms and molecules. Since every dressed molecule contains two atoms, we expect the contribution $2 n_{\mathrm{mc}}$ from the condensate of dressed molecules. Indeed, in the BEC limit it can be shown explicitly that the atomic density $\operatorname{Tr}\left[G_{\mathrm{a}} \sigma_{3}\right]=2 \int d \mathbf{k}\left|v_{\mathrm{a}}(\mathbf{k})\right|^{2} /(2 \pi)^{3}$ contains exactly the expected contribution $2(1-Z) n_{\mathrm{mc}}$ of paired atoms in the Bose-Einstein condensate of dressed molecules. The atomic density does, however, not contain the paired atoms associated with the dressed molecules that are not in the Bose-Einstein condensate. This omission is repaired by the fluctuation corrections which contain both the associated changes in the atomic density and twice the dressed molecular depletion.

We are now in a position to determine $Z$. In principle it is equal to the residue of the pole of $G_{\mathrm{m} ; 11}(\mathbf{0}, i \omega)$ at $\omega=0$. To understand the physics of that result better it is useful to consider the spectral function of the bare molecules with zero momentum, i.e., $\rho_{\mathrm{m}}(\mathbf{0}, \omega)=-\operatorname{Im}\left[G_{\mathrm{m} ; 11}(\mathbf{0}, \omega+\right.$ $i 0)] / \pi$. This spectral function is closely related to the 
density of states of the molecules at zero momentum and thus gives detailed information on the dressed molecular content of the gas. Most importantly for our purpose, the Bose-Einstein condensate of dressed molecules gives rise to a delta function in the spectral function exactly at zero frequency. The strength of this delta function is precisely $Z$, because this is the probability to take a bare molecule out of the Bose-Einstein condensate of dressed molecules. Besides this bound-state contribution, the spectral function contains also a contribution from the continuum of atomic scattering states. In the BEC limit of the crossover the continuum contribution only occurs at positive frequency and starts at a frequency of $-2 \mu / \hbar \simeq 2 \hbar / m a^{2}$ as shown in Fig. 1(a). In the BCS limit the continuum contribution occurs both at positive and negative frequencies, which start at a frequency of about $\pm 2 g \sqrt{Z n_{\mathrm{mc}}} / \hbar$, respectively, due to the gap that exists for the creation of an atomic quasiparticle-quasihole pair. This is shown in Fig. 1(b). The negative frequency part of the spectral function is especially important, because it determines the depletion of the Bose-Einstein condensate of dressed molecules. Physically it represents the dressed molecules that are stabilized by the Fermi sea [27]. Making use of the above physical picture, we finally obtain the desired result,

$$
Z=\frac{1-\Sigma_{11}^{(1)}}{\left(1-\Sigma_{11}^{(1)}\right)^{2}+\Sigma_{12}\left(\Sigma_{12}^{(2)}-\Sigma_{11}^{(2)}\right)},
$$

where $\Sigma_{i j}^{(n)} \equiv(-i)^{n} \partial^{n} \sum_{i j}(\mathbf{0}, 0) / \partial \omega^{n}$. If $n_{\mathrm{mc}}$ vanishes, this exactly equals the two-body $Z$ [11].

At nonzero momenta the spectral function is similar but now contains two delta functions at the frequencies $\pm \omega_{\mathrm{m}}(\mathbf{k})$, which gives us the dispersion for the molecular excitations. This shows how our theory at long wavelengths reduces to a renormalized Bogoliubov theory. One delta function corresponds to a particlelike excitation,
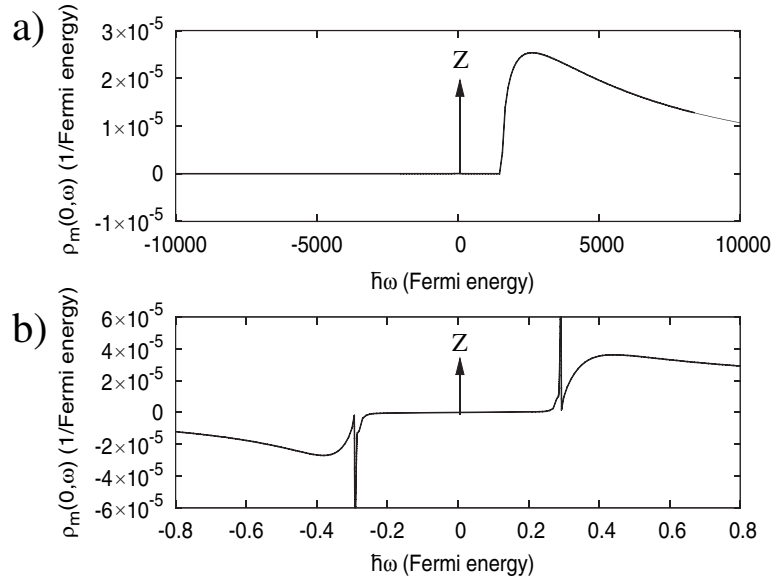

FIG. 1. Spectral functions of the bare ${ }^{6} \mathrm{Li}_{2}$ molecules with zero momentum (a) in the BEC limit at $571 \mathrm{G}$ and (b) in the BCS limit at $892 \mathrm{G}$ of the crossover occurring near the broad Feshbach resonance of ${ }^{6} \mathrm{Li}$ at $834 \mathrm{G}$. The Fermi energy of the gas is $380 \mathrm{nK}$. and is proportional to $\left|u_{\mathrm{m}}(\mathbf{k})\right|^{2}$; the other corresponds to a holelike excitation, and is proportional to $\left|v_{\mathrm{m}}(\mathbf{k})\right|^{2}$. Both are renormalized by $Z$, so by definition the delta functions have strength $Z\left|u_{\mathrm{m}}(\mathbf{k})\right|^{2}$ and $-Z\left|v_{\mathrm{m}}(\mathbf{k})\right|^{2}$, respectively, with $\left|u_{\mathrm{m}}(\mathbf{k})\right|^{2}-\left|v_{\mathrm{m}}(\mathbf{k})\right|^{2}=1$. In the zero-momentum limit these two delta functions result in a single delta function with strength $Z$. Moreover, in agreement with the Goldstone theorem, the quasiparticle dispersion $\omega_{\mathrm{m}}(\mathbf{k})$ always turns out to be linear at long wavelengths [28]. In the following, we therefore determine also the associated speed of (second) sound throughout the BECBCS crossover region.

Results and discussion. - In Fig. 2 we show our results for $Z$ and compare with the experimental data of Partridge et al. [10]. In general the agreement is satisfactory. This is particularly true at relatively low magnetic fields where $Z$ is determined by two-body physics, which is exactly incorporated into our theory. At higher magnetic fields the theoretical values of $Z$ are somewhat higher than the experimental ones. We believe that the reasons for this are twofold. First, the experiment is performed in an optical trap. As a result, the experimental data involve an appropriate average over the density profile of the gas, which lowers the observed value of $Z$. Second, the excited molecular state used in the experiment has a width which is much larger than the Fermi energy of the gas. The measurement, therefore, has not sufficient resolution to probe only the Bose-Einstein condensate of dressed molecules, and probes also the dressed molecules which are not BoseEinstein condensed. This second effect should be especially important at high magnetic fields. To disentangle these different effects, however, goes beyond the scope of

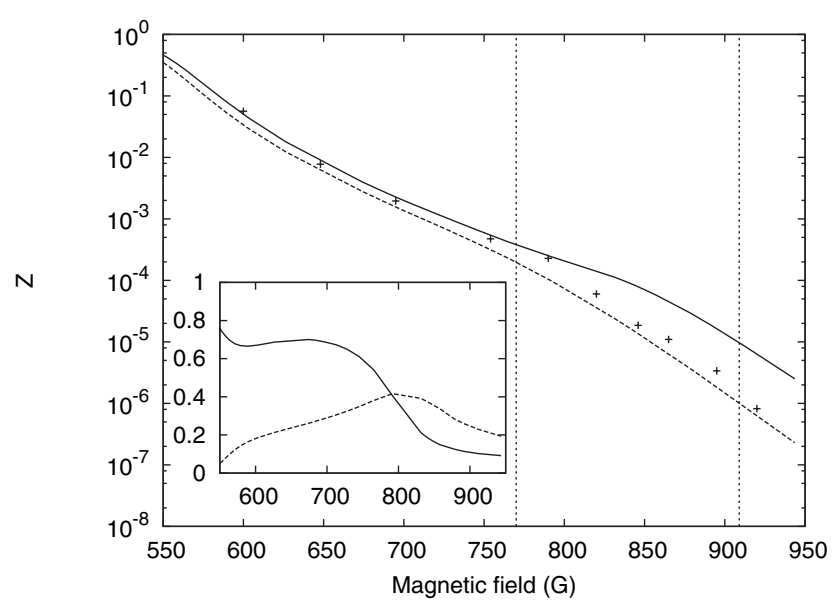

FIG. 2. The solid curve shows the probability $Z$. The data points are from the experiment of Partridge et al. [10]. The dashed curve shows for reference the fraction $2 Z n_{\mathrm{mc}} / n$ as a function of magnetic field. The Fermi energy $\hbar k_{F} / 2 m$ of the gas is $380 \mathrm{nK}$. In the inset the solid line shows the Bose-Einestin condensate fraction of dressed molecules $2 n_{\mathrm{mc}} / n$ and the dashed line the contribution of the fluctuations to the total atomic density. The vertical lines indicate the magnetic fields where $k_{F}|a|=1$. 


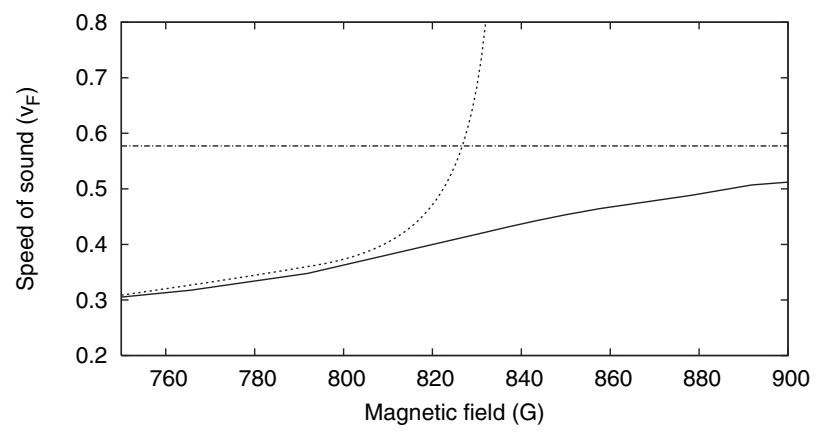

FIG. 3. The speed of sound as a function of magnetic field is given by the solid line. The Fermi energy of the gas is $380 \mathrm{nK}$. The dashed and dash-dotted curves show the Bogoliubov result $\hbar \sqrt{2 \pi a n_{\mathrm{mc}}} / m$ and the speed of sound $\hbar k_{F} / \sqrt{3 m} \equiv v_{F} \sqrt{3}$ of the Anderson-Bogoliubov mode, respectively.

the present Letter and is left for further investigation. Furthermore, to keep a consistent picture of the crossover, all our calculations are done at the same density, since the small changes in $Z$ that result from the changing density are of a smaller order than those due to the inhomogeneity of the trap.

We also show in Fig. 2 the Bose-Einstein condensate fraction of dressed molecules $2 n_{\mathrm{mc}} / n$ throughout the BECBCS crossover. In qualitative agreement with the poor man's approach of Ref. [12], the latter fraction is always substantial below the Feshbach resonance and becomes negligible only sufficiently far above the Feshbach resonance when $k_{F}|a|<1$. This is an important observation, because in our theoretical description of the experiment of Partridge et al. the molecular probe couples to the dressed molecules, which act as distinct entities in the gas since the atom-molecule coupling is much larger than the coupling of the probe laser to the bare molecules. In this manner it is most easy to understand the experimental observation that there is initially an exponential (one-body) decay of a large part of the total atomic density with a rate that is much smaller than the bare molecular photodissociation rate.

For completeness we give in Fig. 2 also the BoseEinstein condensate fraction of bare molecules $2 Z n_{\mathrm{mc}} / n$ [29] and, in the inset, the fluctuation corrections to the total atomic density. As expected, the fluctuation corrections are very important in the crossover region and become small far away from the Feshbach resonance, where mean-field theory applies. From the fluctuations we also extract the speed of sound of the gas, which is shown in Fig. 3. Note that in the BEC limit the speed of sound depends explicitly on the condensate density of dressed molecules $n_{\mathrm{mc}}$, which shows the physical significance of this quantity. In the BCS limit the Anderson-Bogoliubov mode is recovered. In combination with the presence of the sharp peaks in the spectral function in Fig. 1(b), this shows that also the decoupling of the amplitude and phase fluctuations of the Bose-Einstein condensate of dressed molecules that occurs in the BCS limit is correctly incorporated. We therefore conclude that the RPA-like atom-molecule theory devel- oped here gives an excellent account of the subtle interplay between two-body and many-body physics taking place at the crossover near a Feshbach resonance.

We are very grateful for many helpful remarks and stimulating discussions with Randy Hulet.

[1] H. T. C. Stoof, M. Houbiers, C. A. Sackett, and R. G. Hulet, Phys. Rev. Lett. 76, 10 (1996).

[2] E. Timmermans, K. Furuya, P. W. Milonni, and A. K. Kerman, Phys. Lett. A 285, 228 (2001).

[3] Y. Ohashi and A. Griffin, Phys. Rev. Lett. 89, 130402 (2002).

[4] J. N. Milstein, S. J. J. M. F. Kokkelmans, and M. J. Holland, Phys. Rev. A 66, 043604 (2002).

[5] C. A. Regal, M. Greiner, and D. S. Jin, Phys. Rev. Lett. 92, 040403 (2004).

[6] M. W. Zwierlein et al., Phys. Rev. Lett. 92, 120403 (2004).

[7] J. Kinast et al., Phys. Rev. Lett. 92, 150402 (2004); Science 307, 1296 (2005).

[8] M. Bartenstein et al., Phys. Rev. Lett. 92, 120401 (2004); 92, 203201 (2004).

[9] T. Bournel et al., Phys. Rev. Lett. 93, 050401 (2004).

[10] G. B. Partridge et al., Phys. Rev. Lett. 95, 020404 (2005).

[11] For a recent review, see R. A. Duine and H. T. C. Stoof, Phys. Rep. 396, 115 (2004).

[12] G. M. Falco and H.T.C. Stoof, Phys. Rev. Lett. 92, 130401 (2004).

[13] A. Perali, P. Pieri, L. Pisani, and G. C. Strinati, Phys. Rev. Lett. 92, 220404 (2004).

[14] J. Stajic, Q. Chen, and K. Levin, Phys. Rev. Lett. 94, 060401 (2005).

[15] M. Mackie and J. Piilo, Phys. Rev. Lett. 94, 060403 (2005).

[16] R. B. Diener and T.-L. Ho, cond-mat/0404517.

[17] Y. Ohashi and A. Griffin, Phys. Rev. A 72, 013601 (2005).

[18] M. H. Szymanska, K. Goral, T. Koehler, and K. Burnett, Phys. Rev. A 72, 013610 (2005).

[19] S. Diehl and C. Wetterich, cond-mat/0502534.

[20] G.E. Astrakharchik, R. Combescot, X. Leyronas, S. Stringari, Phys. Rev. Lett. 95, 030404 (2005).

[21] P.D. Drummond, K. V. Kheruntsyan, and H. He, Phys. Rev. Lett. 81, 3055 (1998).

[22] E. Timmermans, P. Tommasini, H. Hussein, and A. Kerman, Phys. Rep. 315, 199 (1999).

[23] M. Houbiers, H. T. C. Stoof, W. I. McAlexander, and R. G. Hulet, Phys. Rev. A 57, R1497 (1998).

[24] K. M. O'Hara et al., Phys. Rev. A 66, 041401(R) (2002).

[25] G. M. Falco and H. T. C. Stoof, Phys. Rev. A 71, 063614 (2005).

[26] N. M. Hugenholtz and D. Pines, Phys. Rev. 116, 489 (1959).

[27] G. M. Falco, R. A. Duine, and H. T. C. Stoof, Phys. Rev. Lett. 92, 140402 (2004).

[28] Y. Ohashi and A. Griffin, Phys. Rev. A 67, 063612 (2003).

[29] Recently, we became aware of other work by Q. Chen and K. Levin, this issue, Phys. Rev. Lett. 95, 260406 (2005) and J. Javanainen, M. Kostrun, M. Mackie, and A. Carmichael, cond-mat/0506183 that also calculate this quantity, but without fluctuation corrections. 